\title{
Mitochondrial Dysfunction, Endoplasmic Reticulum Stress, and Apoptosis in Alzheimer's Disease
}

\author{
Kazuhiro Takuma ${ }^{1, *}$, Shirley ShiDu Yan ${ }^{2}$, David M. Stern ${ }^{3}$, and Kiyofumi Yamada ${ }^{1}$ \\ ${ }^{1}$ Laboratory of Neuropsychopharmacology, Graduate School of Natural Science and Technology, Kanazawa University, \\ Kakuma-machi, Kanazawa 920-1192, Japan \\ ${ }^{2}$ Departments of Surgery and Pathology, College of Physicians and Surgeons, Columbia University, \\ New York, NY 10032, USA \\ ${ }^{3}$ Dean's Office, Medical College of Georgia, Augusta, GA 30912, USA
}

Received December 17, 2004

\begin{abstract}
Alzheimer's disease (AD) is the most common neurodegenerative disorder of late life characterized by insidious, chronic, and progressive memory impairment in association with the accumulation of senile plaques, neurofibrillary tangles, and massive loss of neurons. Apoptosis is believed to be an important contributor to progression and pathology of neurodegeneration in $\mathrm{AD}$. There is considerable evidence that amyloid $\beta$-peptide, a major component of senile plaques, has the capacity to activate intracellular apoptosis pathways leading to neuronal cell death. AD-related mutations in genes coding presenilins are also shown to cause neuronal apoptosis, by directly and indirectly regulating apoptotic signaling cascades. Recent evidence suggests that two intrinsic pathways, mitochondrial dysfunction and endoplasmic reticulum stress, are central in the execution of apoptosis in AD. This review summarizes recent progress of research in this field focused on the molecular mechanisms involved in neuronal apoptosis mediated by organelle dysfunction.
\end{abstract}

Keywords: Alzheimer's disease, apoptosis, mitochondrial dysfunction, endoplasmic reticulum stress, amyloid $\beta$-peptide

\section{Introduction}

Alzheimer's disease (AD) is a progressive neurodegenerative disease of the central nervous system associated with nonrelenting deterioration of cognition and memory, resulting in dementia (1). Two pathological hallmarks are observed in AD patients at autopsy: intracellular neurofibrillary tangles and extracellular senile plaques in the neocortex, hippocampus, and other subcortical regions of the brain essential for cognitive function (2). Neurofibrillary tangles are formed from paired helical filaments composed of neurofilaments and hyperphosphorylated tau protein. Plaque cores are formed mostly from deposition of amyloid $\beta$-peptide (A $\beta)$, a $39-43$-amino acid peptide

*Corresponding author. FAX: +81-76-234-4416

E-mail: ktakuma@p.kanazawa-u.ac.jp

Invited article derived by cleavage of the amyloid precursor protein (APP), although there are other associated proteins /proteoglycans. Several lines of evidence indicate that fibrillar $\mathrm{A} \beta$ and, especially, soluble $\mathrm{A} \beta$ aggregates are important in the etiology of AD. Neurotoxicity mediated by $\mathrm{A} \beta$ has been well established both in vivo and in vitro, and $\mathrm{A} \beta$-induced neuronal death, based primarily on results of in vitro studies, has been shown to involve oxidative stress, perturbation of intracellular calcium homeostasis, and activation of apoptotic pathways (2). In addition, studies of postmortem brain tissue indicate that neuronal apoptosis occurs in AD (3). Thus, identification of molecular and cellular mechanisms that predispose neurons to apoptotic death in $\mathrm{AD}$ may provide defined targets for new treatment strategies for earlier diagnosis, prevention or ameliorating the course of AD. This review summarizes current knowledge of the "apoptotic phenomenon" in AD and focuses on molecular mechanisms, especially mitochondrial dys- 
function and endoplasmic reticulum (ER) stress.

\section{Apoptosis in neurodegeneration}

Neuronal death occurs by necrosis or apoptosis, which differ morphologically and biochemically. Necrosis is the result of extreme perturbation of the cellular environment (i.e., cellular accidents), as occurs in ischemic insults or trauma. Because intracellular constituents leak out into the extracellular space, a considerable degree of inflammation often accompanies necrosis. In contrast, apoptosis, also known as programmed cell death, is dependent on intracellular pathways resulting in cellular commitment to a defined series of steps resulting in cell suicide. Apoptosis is an important mechanism in normal cell turnover, in growth and development, as well as in maturity. Exposure of acidic phospholipids on the cell membrane during apoptosis is a signal for phagocytic uptake of apoptotic cells. The latter occurs considerably before loss of membrane integrity and unregulated leakage of intracellular contents. It has been generally believed that the predominant mechanism of pathologic cell death in CNS injury is necrotic, and physiological cell death during brain development is regarded as apoptotic. However, recent accumulating evidence strongly suggests that apoptosis might additionally contribute to neuronal death in a variety of neurodegenerative disorders such as Alzheimer's, Parkinson's, and Huntington's diseases and amyotrophic lateral sclerosis (4).

The execution of neuronal apoptosis involves relatively few pathways that converge on activation of the cysteine proteases called caspases (5). To date, 14 caspases have been identified in mammals. Two principal pathways are well-known with respect to their activation: the cell surface death receptor pathway and the mitochondrial pathway. In the death receptor pathway, activation of caspase- 8 is the critical event that transmits the death signal. In the mitochondrial pathway, caspase activation is triggered by the formation of an apoptotic protease-activating-factor-1 (Apaf-1)/cytochrome $c$ complex that is fully functional in recruiting and activating procaspase-9. Activated caspase-9 then cleaves and activates downstream caspases, such as caspase-3, -6, and -7 . The mitochondrial pathway appears to be regulated by the Bcl-2 family of proteins, and there may be participation of ion channels/nonselective pores, in particular the permeability transition pore (PTP), that may be activated by pro-apoptotic stimuli. In addition, recent studies indicate that the ER can sense and transduce apoptotic signals (6). ER stress activates caspase12, thereby triggering an ER-stress-specific cascade for implementation of apoptosis. Accumulating evidence implicates selective neuronal loss in neurodegenerative diseases, including $\mathrm{AD}$, which involves activation of these caspases.

\section{Mitochondrial dysfunction in Alzheimer's disease}

Mitochondria play a central role in both cell life and death (7). Mitochondria are the essential for production of metabolic energy, ATP, through oxidative phosphorylation. Mitochondria also regulate intracellular $\mathrm{Ca}^{2+}$ homeostasis and are the principal generators of intracellular reactive oxygen species (ROS). In contrast, mitochondria also play a key role in controlling pathways that lead to apoptosis. Defects of mitochondrial function can result in excessive production of ROS, formation of the PTP, and release of small proteins that trigger the initiation of apoptosis, such as cytochrome $c$ and apoptosis-inducing factor (AIF), from the mitochondrial intermembrane space into the cytoplasm. Released cytochrome $c$ binds Apaf-1 and activates the caspase cascade (8). Such mitochondrial dysfunction has been proposed as a potential mechanism in the development and pathogenesis of $\mathrm{AD}$, and neuronal apoptosis has been detected in AD brain.

More direct evidence for mitochondrial dysfunction in AD comes from several reports of cytochrome $c$ oxidase deficiency in AD brain (9). Histochemical analyses reveal significant reduction of cytochrome $c$ oxidase activity in the dentate gyrus and other subfields of the hippocampus of $\mathrm{AD}$ patients. In situ hybridization studies also show decreased mRNA levels of the mitochondrial DNA (mtDNA)-encoded subunit II, but not the nuclear DNA-encoded subunit IV, of cytochrome $c$ oxidase in AD brain. High levels of mtDNA mutations, linked to cytochrome $c$ oxidase deficiency, are observed in more frequently in hippocampal pyramidal neurons of AD patients, compared to age-matched controls (10). Human teratocarcinoma cells expressing mtDNA from $\mathrm{AD}$ subjects display reduced cytochrome $c$ oxidase activity, elevated ROS, and reduced ATP levels, compared with the cells expressing mtDNA from agematched control subjects (11). These observations suggest that alterations in mtDNA may play a key role in mitochondrial dysfunction in AD. Furthermore, current evidence indicates $\mathrm{A} \beta$ may cause mitochondrial dysfunction, resulting in oxidative stress and caspase activation (12). In isolated mitochondria, $\mathrm{A} \beta$ inhibits activities of mitochondrial electron transport chain complexes, $\alpha$-ketoglutarate dehydrogenase, pyruvate dehydrogenase, and cytochrome $c$ oxidase, and promotes $\mathrm{Ca}^{2+}$-induced assembly of the PTP. A $\beta$ fragment 25-35 $\left(\mathrm{A} \beta_{25-35}\right)$ and $\mathrm{A} \beta_{1-42}$ cause a loss of mitochondrial membrane potential by activating NADPH 
oxidase, as well as metabolic pathways upstream of mitochondrial respiration, in astrocytes (13). $\mathrm{A} \beta_{25-35}$ inhibits respiratory complex I and decreases cellular ATP content of both astrocytes and neurons (14). A $\beta_{25-}$ 35 induces translocation of the second-mitochondriaderived activator of caspase (Smac) from mitochondria to cytosol via AP-1/Bim activation (15). Intracellular $\mathrm{A} \beta_{1-42}$ selectively causes apoptosis in human neurons through p53 and Bax; the latter activates caspases by promoting release of mitochondrial cytochrome $c$ (16). Expression of a mutant form of APP (mAPP) into PC12 cells and human embryonic kidney cells results in substantial elevation of $\mathrm{A} \beta$ levels and is associated with increased levels of nitric oxide (NO) and reduced ATP, finally leading to cell death (17). DAPT $(N-[N-(3,5-$ difluorophenacetyl)-L-alanyl]-S-phenylglycine $t$-butyl ester), a functional $\gamma$-secretase inhibitor, decreases intracellular $\mathrm{A} \beta$ production and normalizes NO and ATP levels in the cells expressing mAPP. These findings lead to the proposal that $\mathrm{A} \beta$ directly disrupts mitochondrial function and may contribute to the deficiency of energy metabolism and neuronal apoptosis seen in AD. Moreover, recent data indicate that $\mathrm{A} \beta$ interacts with a binding protein, termed $\mathrm{A} \beta$-binding alcohol dehydrogenase (ABAD), in mitochondria and directly causes mitochondrial dysfunction $(18,19)$. Neurons cultured from transgenic mice overexpressing $\mathrm{mAPP}$ and $\mathrm{ABAD}$ display spontaneous generation of ROS, loss of mitochondrial membrane potential and decreased ATP, as well as subsequent release of cytochrome $c$ from mitochondria and induction of caspase-3-like activity followed by apoptotic cell death. Consistent with these results, a decoy peptide that blocks $\mathrm{A} \beta$-ABAD interaction prevents the mitochondrial dysfunction and apoptosis in an $\mathrm{A} \beta$-rich environment in vitro.

In summary, mitochondrial dysfunction and the resulting energy deficit may trigger the onset of neuronal apoptosis in $\mathrm{AD}$.

\section{ER stress in Alzheimer's disease}

The ER is a multifaceted organelle that regulates protein synthesis, protein folding and trafficking, cellular responses to stress, and intracellular $\mathrm{Ca}^{2+}$ levels (20). Disruption of $\mathrm{Ca}^{2+}$ homeostasis, inhibition of protein $\mathrm{N}$-linked glycosylation, expression of mutant proteins, and various other types of cellular stresses cause accumulation of misfolded proteins in the ER lumen, resulting in ER stress (21). ER stress elicits an adaptive response triggering a signaling pathway called the unfolded protein response (UPR), which alleviates stress by induction of ER-localized chaperones, initiation of a degradation system and attenuation of protein synthesis. However, if the stress is prolonged, it may lead to processing of the ER-resident protease procaspase-12 as well as activation of calpain, caspase-3, caspase-6, and apoptosis. Recent evidence suggests that ER stress-mediated apoptotic signal pathways may contribute to the pathogenesis of $\mathrm{AD}$ (22).

AD-linked mutations in presenilins (PS), two closely related polytopic integral membrane proteins (PS-1 and PS-2) that reside in the ER, appear to impair the ER stress response and enhance vulnerability to stressinduced apoptosis in part by altering proteolytic processing of APP. PS- 1 mutations deregulate neuronal $\mathrm{Ca}^{2+}$ homeostasis by direct attenuation of capacitative $\mathrm{Ca}^{2+}$ entry at the cell-surface, independent of APP, and indirectly by increasing $\mathrm{Ca}^{2+}$ release from the ER via processing of APP and generation of amyloid peptides. Such perturbation of $\mathrm{Ca}^{2+}$ homeostasis causes apoptosis by activation of the $\mathrm{Ca}^{2+}$-dependent apoptotic execution proteases. PS-1 mutations sensitize neurons to DNA damage-induced death by promoting ER-mediated apoptotic proteolytic cascades such as activation of calpains and caspase-12 (23). A familial AD-associated mutation in PS-2, M239I, also alters cellular $\mathrm{Ca}^{2+}$ homeostasis in two cell lines (HEK293 and HeLa) following transfection (24). These data are consistent with evidence that $\mathrm{Ca}^{2+}$ release from the ER is significantly reduced in fibroblasts from familial AD patients, compared to cells from healthy individuals. In addition to $\mathrm{Ca}^{2+}$ dysregulation, PS mutations down-regulate the UPR and enhance vulnerability to ER stress (25). PS1 mutations interfere with the signaling pathways of the UPR by interfering with function of IRE1, a stresssensor in the ER lumen, decreasing expression of GRP78/Bip, an ER molecular chaperone, and interfering with activation of PERK, an ER stress transducer. The UPR is reduced in PS- 1 knockout cells, with a reduction in nuclear accumulation of IRE1. PS1 mutations have also been shown to induce a pro-apoptotic factor in the ER involved in stress-mediated apoptotic pathways, GADD153/CHOP (26). PS is a component of the $\gamma$-secretase complex, which, together with $\beta$ secretase, cleaves APP and produces $\mathrm{A} \beta$, and PS mutations increase production of total $\mathrm{A} \beta$ and $\mathrm{A} \beta_{1-42}$ (27). Mutant PS1 selectively increase $\mathrm{A} \beta_{1-42}$ secretion when $N$-glycosylation is impaired. Herp, an ER stressinducible membrane protein, enhances PS-mediated $\mathrm{A} \beta$ generation, possibly through its binding to PS (28).

It has been proposed that $\mathrm{A} \beta$ can directly mediate $\mathrm{ER}$ stress responses and apoptosis, because $\mathrm{A} \beta$ is synthesized and accumulates in the ER. A $\beta_{1-42}$ activates caspase-12 in primary neurons through calpain activation, and caspase- 12 knockout neurons are partially resistant to $\mathrm{A} \beta$-induced cell death (29). $\mathrm{A} \beta$ has also 
Mitochondria

mtDNA mutations

ABAD-A $\beta$ interaction

Decreased cyt $c$ oxidase activity

ROS generation

Loss of membrane potential

ATP depletion

PTP formation

Release of cyt $c$ and Smac

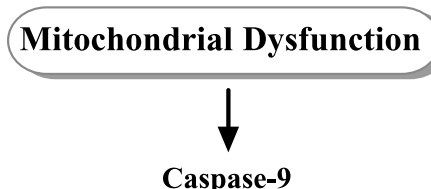

activation

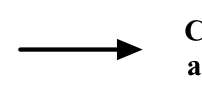

Caspase-3
activation

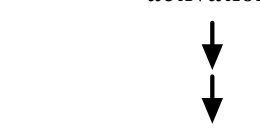

Neuronal Apoptosis

\section{Endoplasmic reticulum}

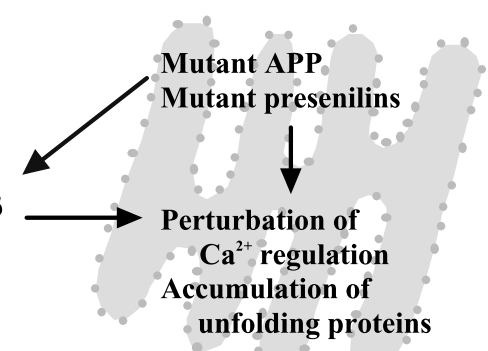

ER Stress

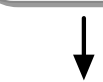

Caspase-12 activation

Fig. 1. Possible signal pathways of neuronal apoptosis in Alzheimer's disease (AD). Mutant APP (amyloid precursor protein) and presenilins in endoplasmic reticulum (ER) accelerate the formation of amyloid $\beta$-protein $(\mathrm{A} \beta)$. The increased $\mathrm{A} \beta$ causes mitochondrial dysfunction and/or ER stress, resulting in the activation of apoptotic signal pathways, including caspases, which underlie neuronal death in AD. mtDNA: mitochondrial DNA, ABAD: A $\beta$-binding alcohol dehydrogenase, cyt $c$ : cytochrome $c$, ROS: reactive oxygen species, PTP: permeability transition pore, Smac: second-mitochondria-derived activator of caspase.

been shown to trigger $\mathrm{Ca}^{2+}$ release from the ER and induce ER stress and neurotoxicity (30).

Thus, ER stress is a key event in triggering and mediating neuronal death in AD. However, further study is needed to clarify the molecular mechanisms underlying the ER stress-mediated neuronal apoptosis.

\section{Conclusion}

There is accumulating evidence from in vitro, in vivo, and human studies suggesting that apoptosis is likely to have an essential role in pathogenesis of AD. Thus, identification of the apoptotic mechanisms operative in AD may provide insights into potential future therapeutic strategies for AD. Here, we propose that two major pathways are important in $\mathrm{AD}$-associated apoptosis: mitochondrial dysfunction- and ER stressmediated pathways (Fig. 1). In particular, increased $\mathrm{A} \beta$, one of the etiological factors in $\mathrm{AD}$, appears to have a central role in neuronal apoptosis, because it can activate both cell-death pathways. Familial AD-linked mutations in PS, which resides in ER and increases $\mathrm{A} \beta$ production, also cause apoptosis. These finding suggest that ER stress and mitochondrial dysfunction might cooperatively regulate apoptotic signaling cascades. However, the precise molecular mechanisms underlying mito- chondrial dysfunction and ER stress in neuronal cell death and neurodegeneration remain unknown. More work in this area may lead to the discovery of novel therapeutic targets for AD.

\section{Acknowledgments}

This study was supported in part by a grant for the 21 st Century COE Program from the Ministry of Education, Culture, Sports, Science, and Technology of Japan, a Grant-in-Aid for Scientific Research from the Japan Society for the Promotion of Science, and an Astra Zeneca Research Grant.

\section{References}

1 Selkoe DJ. Alzheimer's disease: genes, proteins, and therapy. Physiol Rev. 2001;81:741-766.

2 Mattson MP. Pathways towards and away from Alzheimer's disease. Nature. 2004;430:631-639.

3 Colurso GJ, Nilson JE, Vervoort LG. Quantitative assessment of DNA fragmentation and $\beta$-amyloid deposition in insular cortex and midfrontal gyrus from patients with Alzheimer's disease. Life Sci. 2003;73:1795-1803.

4 Mattson MP. Apoptosis in neurodegenerative disorders. Nat Rev Mol Cell Biol. 2000;1:120-129.

5 Friedlander RM. Apoptosis and caspases in neurodegenerative 
diseases. N Engl J Med. 2003;348:1365-1375.

6 Breckenridge DG, Germain M, Mathai JP, Nguyen M, Shore GC. Regulation of apoptosis by endoplasmic reticulum pathways. Oncogene. 2003;22:8608-8618.

7 Duchen MR. Mitochondria in health and disease: perspectives on a new mitochondrial biology. Mol Aspects Med. 2004;25: 365-451.

8 Hengartner MO. The biochemistry of apoptosis. Nature. 2000; 407:770-776.

9 Ojaimi J, Byrne E. Mitochondrial function and Alzheimer's disease. Biol Signals Recept. 2001;10:254-262.

10 Cottrell DA, Borthwick GM, Johnson MA, Ince PG, Turnbull DM. The role of cytochrome $c$ oxidase deficient hippocampal neurones in Alzheimer's disease. Neuropathol Appl Neurobiol. 2002;28:390-396.

11 Cardoso SM, Santana I, Swerdlow RH, Oliveira CR. Mitochondria dysfunction of Alzheimer's disease cybrids enhances A $\beta$ toxicity. J Neurochem. 2004;89:1417-1426.

12 Eckert A, Keil U, Marques CA, Bonert A, Frey C, Schussel K, et al. Mitochondrial dysfunction, apoptotic cell death, and Alzheimer's disease. Biochem Pharmacol. 2003;66:1627-1634.

13 Abramov AY, Canevari L, Duchen MR. $\beta$-Amyloid peptides induce mitochondrial dysfunction and oxidative stress in astrocytes and death of neurons through activation of NADPH oxidase. J Neurosci. 2004;24:565-575.

14 Casley CS, Land JM, Sharpe MA, Clark JB, Duchen MR, Canevari L. $\beta$-Amyloid fragment $25-35$ causes mitochondrial dysfunction in primary cortical neurons. Neurobiol Dis. 2002; 10:258-267.

15 Yin KJ, Lee JM, Chen SD, Xu J, Hsu CY. Amyloid- $\beta$ induces Smac release via AP-1/Bim activation in cerebral endothelial cells. J Neurosci. 2002;22:9764-9770.

16 Zhang Y, McLaughlin R, Goodyer C, LeBlanc A. Selective cytotoxicity of intracellular amyloid $\beta$ peptide $_{1-42}$ through $\mathrm{p} 53$ and Bax in cultured primary human neurons. J Cell Biol. 2002;156:519-529.

17 Keil U, Bonert A, Marques CA, Scherping I, Weyermann J, Strosznajder JB, et al. Amyloid $\beta$-induced changes in nitric oxide production and mitochondrial activity lead to apoptosis. J Biol Chem. 2004;279:50310-50320.

18 Lustbader JW, Cirilli M, Lin C, Xu HW, Takuma K, Wang N, et al. ABAD directly links $\mathrm{A} \beta$ to mitochondrial toxicity in Alzheimer's disease. Science. 2004;304:448-452.
19 Takuma K, Yao J, Huang J, Xu H, Chen X, Luddy J, et al. ABAD enhances $\mathrm{A} \beta$-induced cell stress via mitochondrial dysfunction. FASEB J. In press 2005.

20 Rao RV, Ellerby HM, Bredesen DE. Coupling endoplasmic reticulum stress to the cell death program. Cell Death Differ. 2004;11:372-380.

21 Kaufman RJ. Stress signaling from the lumen of the endoplasmic reticulum: coordination of gene transcriptional and translational controls. Genes Dev. 1999;13:1211-1233.

22 Katayama T, Imaizumi K, Manabe T, Hitomi J, Kudo T, Tohyama M. Induction of neuronal death by ER stress in Alzheimer's disease. J Chem Neuroanat. 2004;28:67-78.

23 Chan SL, Culmsee C, Haughey N, Klapper W, Mattson MP. Presenilin-1 mutations sensitize neurons to DNA damageinduced death by a mechanism involving perturbed calcium homeostasis and activation of calpains and caspase-12. Neurobiol Dis. 2002;11:2-19.

24 Zatti G, Ghidoni R, Barbiero L, Binetti G, Pozzan T, Fasolato C, et al. The presenilin 2 M239I mutation associated with familial Alzheimer's disease reduces $\mathrm{Ca}^{2+}$ release from intracellular stores. Neurobiol Dis. 2004;15:269-278.

25 Imaizumi K, Miyoshi K, Katayama T, Yoneda T, Taniguchi M, Kudo T, et al. The unfolded protein response and Alzheimer's disease. Biochim Biophys Acta. 2001;1536:85-96.

26 Milhavet O, Martindale JL, Camandola S, Chan SL, Gary DS, Cheng A, et al. Involvement of Gadd153 in the pathogenic action of presenilin-1 mutations. J Neurochem. 2002;83:673681.

27 Lleo A, Berezovska O, Growdon JH, Hyman BT. Clinical, pathological, and biochemical spectrum of Alzheimer disease associated with PS-1 mutations. Am J Geriatr Psychiatry. 2004; 12:146-156.

28 Sai X, Kawamura Y, Kokame K, Yamaguchi H, Shiraishi H, Suzuki R, et al. Endoplasmic reticulum stress-inducible protein, Herp, enhances presenilin-mediated generation of amyloid $\beta$ protein. J Biol Chem. 2002;277:12915-12920.

29 Nakagawa T, Yuan J. Cross-talk between two cysteine protease families. Activation of caspase-12 by calpain in apoptosis. J Cell Biol. 2000;150:887-894.

30 Suen KC, Lin KF, Elyaman W, So KF, Chang RC, Hugon J. Reduction of calcium release from the endoplasmic reticulum could only provide partial neuroprotection against $\beta$-amyloid peptide toxicity. J Neurochem. 2003;87:1413-1426. 